\title{
Efeito do armazenamento de argilas esmectíticas nas suas propriedades reológicas
}

\section{(Effect of smectite clays storage in their rheological properties)}

\author{
I. A. da Silva', F. K. A. de Sousa ${ }^{2}$, H. S. Ferreira ${ }^{3}$, H. S. Ferreira ${ }^{4}$, G. de A. Neves ${ }^{5}$, H. C. Ferreira \\ ${ }^{1}$ Programa de Pós-Graduação em Ciência e Engenharia de Materiais, ${ }^{2}$ Unidade Acadêmica de Engenharia \\ de Produção, ${ }^{5}$ Unidade Acadêmica de Engenharia de Materiais, Universidade Federal de Campina Grande, \\ Campina Grande, PB, Brasil 58109-970 \\ ${ }^{3}$ Departamento de Educação, Universidade Federal Rural de Pernambuco, Recife, PE, Brasil 52171-130 \\ ${ }^{4}$ Departamento de Engenharia de Materiais, Universidade Federal da Paraíba, João Pessoa, PB, Brasil 58051-900 \\ isabelle_albuquerquecg@hotmail.com,kegalves@gmail.com,hsivini@terra.com.br,heber@ct.ufpb.br, \\ gelmires.neves@ufcg.edu.br,heber.ferreira@ufcg.edu.br
}

\begin{abstract}
Resumo
Este trabalho investigou a influência do armazenamento de argilas esmectíticas naturais e industrializadas nas suas propriedades reológicas, tendo em vista que a reação de dupla troca que ocorre após o tratamento das argilas policatiônicas com o $\mathrm{Na}_{2} \mathrm{CO}_{3}$ é reversível. Os fenômenos envolvidos nesta reação ainda não são totalmente conhecidos e estudos anteriores mostram melhoras em algumas propriedades. As propriedades reológicas foram determinadas em argilas sódicas em 1995 e policatiônicas aditivadas com carbonato de sódio $\left(\mathrm{Na}_{2} \mathrm{CO}_{3}\right)$ em 2015. A caracterização física, química e mineralógica das amostras foi realizada através das seguintes técnicas: análise granulométrica por difração a laser, composição química por fluorescência de raios X, difração de raios X e análises térmicas (DTA e TG). A reologia das dispersões foi determinada através da viscosidade aparente, viscosidade plástica e volume do filtrado, onde posteriormente foram consideradas normas da indústria do petróleo apenas como parâmetro de referência. Os resultados mostraram que as condições de armazenamento, umidade e tamanho de partículas das amostras trouxeram melhoras nas suas propriedades reológicas no decorrer dos anos, indicando a não reversibilidade da reação de troca de cátions, o que é importante na sua validade após fabricação.

Palavras-chave: argilas esmectíticas, armazenamento, envelhecimento, propriedades reológicas, fluidos de perfuração.
\end{abstract}

\begin{abstract}
This work investigates the storage influence of natural and industrial smectite clays in their rheological properties, since the salt metathesis reaction that occurs following treatment of polycationic clays with $\mathrm{Na}_{2} \mathrm{CO}_{3}$ is reversible. The phenomena involved in this reaction are not yet fully known and previous studies show improvement in some properties. The rheological properties were determined in sodium-clays in 1995 and polycationic clays added with sodium carbonate $\left(\mathrm{Na}_{2} \mathrm{CO}_{3}\right)$ in 2015. Physical, chemical and mineralogical characterizations of the samples were performed using the following techniques: particle size analysis by laser diffraction, chemical composition by X-ray fluorescence, X-ray diffraction and thermal analysis (DTA and TGA). The rheology of dispersions was determined by the apparent viscosity, plastic viscosity and filtrate volume, which were later considered the oil industry standards only as a benchmark. The results showed that the storage conditions, humidity and particle size of the samples resulted in improvements in their rheological properties over the years, indicating the non-reversibility of the reaction of cation exchange, which is important in their validity after manufacturing.
\end{abstract}

Keywords: smectite clays, storage, aging, rheological properties, drilling fluids.

\section{INTRODUÇÃO}

As argilas esmectíticas são argilas plásticas, constituídas essencialmente por argilominerais do grupo da esmectita, especialmente a montmorilonita e minerais acessórios como quartzo, feldspato, dentre outros; são dioctaédricas e apresentam propriedades coloidais bem particulares, como plasticidade e inchamento [1]. Em geral, as argilas esmectíticas brasileiras são policatiônicas e tradicionalmente são transformadas em sódicas através da adição de carbonato de sódio $\left(\mathrm{Na}_{2} \mathrm{CO}_{3}\right)$ [2-4]. A presença das argilas na forma cálcica e policatiônica conduz a resultados reológicos insatisfatórios. No processo de troca de cátions, tradicionalmente os íons de $\mathrm{Na}^{+}$substituem os íns de $\mathrm{Ca}^{2+}$ conduzindo a uma melhor condição no estado de defloculação, resultando em propriedades reológicas adequadas para o uso das argilas esmectíticas sódicas como componente chave nos fluidos de perfuração à base água $[5,6]$. O comportamento reológico de dispersões de argilas é de significativo interesse científico e tecnológico 
em um grande número de processos industriais [7]. Argilas esmectíticas são usadas em lamas de perfuração devido à sua capacidade para formar dispersões tixotrópicas de elevada viscosidade na presença de água, fornecendo as propriedades reológicas necessárias quando em repouso e durante o cisalhamento $[8,9]$.

Estudos sobre envelhecimento e armazenamento/ acondicionamento adequado de argilas esmectíticas $[1,10]$ têm por objetivo verificar a reversibilidade das reações de troca de cátions com consequente perda de propriedades frente aos mais diversos aspectos, fornecendo dados sobre a vida útil ou validade dos produtos industriais [11, 12]. A reversibilidade das reações pode ser verificada de forma indireta pelo controle reológico. Outros fatores como a umidade, a fração argilosa e diferentes processos industriais de aditivação também podem ser capazes de modificar as propriedades reológicas das argilas. Estudo anterior observou que, dependendo do tipo de argila e do processamento industrial a que foram submetidas, as argilas bentoníticas podem apresentar maior ou menor grau de estabilidade em suas propriedades reológicas com o tempo, sendo aquelas com características floculadas-gel as mais susceptíveis ao efeito do envelhecimento. Observaram também que depois de um tempo de envelhecimento de 7 anos, todas as dispersões das argilas apresentaram aumento em seus valores de viscosidade aparente e plástica [13]. Do mesmo modo, outro estudo observou que o armazenamento de argilas, que foram submetidas à ação de elementos ambientais durante um período razoável de tempo (6 meses) antes de entrar no processo de produção, melhorou suas propriedades tecnológicas $[14,15]$. Seguindo essa linha de raciocínio, mais estudos foram feitos, dentre eles foi observado que o envelhecimento e/ou a qualidade da aditivação depende da umidade da argila e da dosagem do sódio no processo de aditivação, onde a umidade da argila deve ser considerada e controlada [11], bem como que os efeitos da concentração e do $\mathrm{pH}$ nas propriedades reológicas de suspensões de bentonitas a longo prazo necessitam de uma maior taxa de cisalhamento nessas suspensões, uma vez que, com o aumento do tempo, há uma maior força de coesão entre as partículas [1]. Outra pesquisa [16] observou que o envelhecimento das argilas variou com o aumento da temperatura e que o cloreto de potássio $(\mathrm{KCl})$ modificou a estabilidade e o comportamento de envelhecimento de suspensões de lamas de perfuração por reduzir a força atrativa das partículas [12].

Com base nestes fatos, observa-se que apesar dos esforços para uma plena compreensão do mecanismo que governa o comportamento do envelhecimento de argilas não é completamente entendido e continua a ser um importante desafio [16, 17], onde os estudos divergem entre fatores químicos, como alterações da carga elétrica da argila com oxidação de compostos inorgânicos, e biológicos, como a oxidação de matéria orgânica. Além destes mecanismos químicos e biológicos, mecanismos físicos são citados como sendo também responsáveis pela melhora das propriedades tecnológicas de argilas durante o envelhecimento, como o aumento da umidade quando as argilas estão estocadas [16, 18]. No ano de 1995, foram estudadas diversas amostras de argilas bentoníticas de Boa Vista, PB, bem como amostras dessas argilas industrializadas pela Bentonit União Nordeste, tendo como objetivo o estudo da influência do CMC na reologia dos fluidos de perfuração base água [19]. Após estes estudos, as amostras ficaram armazenadas em sacos de polietileno até o ano de 2015, ou seja, por vinte anos. Deve ser destacado que os estudos sobre o envelhecimento das esmectitas sódicas industrializadas têm grande importância industrial, pois o prazo de validade geralmente é inferior a um ano, o que pelos dados bibliográficos aqui apresentados é excessivamente reduzido. Desta forma, o objetivo deste trabalho foi verificar a influência do armazenamento de argilas esmectíticas naturais e industrializadas nas suas propriedades reológicas.

\section{MATERIAIS E METODOLOGIA}

Materiais: para esse estudo foram utilizadas 8 amostras de argilas esmectíticas obtidas no ano de 1995 (AM1 a AM8), sendo: i) as 4 primeiras (AM1, AM2, AM3 e AM4) argilas esmectíticas sódicas industrializadas; ii) as 4 últimas (AM5, AM6, AM7 e AM8) argilas esmectíticas policatiônicas naturais. As amostras citadas foram fornecidas pela Bentonit União Nordeste S/A (Campina Grande, Paraíba). Foi utilizado o carbonato de sódio $\mathrm{PA}\left(\mathrm{Na}_{2} \mathrm{CO}_{3}\right)$, pureza de $99,5 \%$ (Labsynth Produtos para Laboratórios Ltda., Diadema, SP).

Metodologia: a caracterização das argilas esmectíticas foi realizada através das técnicas: análise granulométrica $(\mathrm{AG}$, 1064, Cilas), fluorescência de raios X (EDX 720, Shimadzu), difração de raios X (DRX, XRD 6000, Shimadzu) - todas as amostras foram ensaiadas com e sem adição de etileno glicol para confirmar as argilas do grupo da esmectita; as curvas de análise térmica diferencial (DTA) e termogravimetria (TG) foram obtidas através do sistema de análises térmicas (RB-3000, BP Eng. Ind. e Com.), com taxa de aquecimento de $12,5^{\circ} \mathrm{C} / \mathrm{min}$, onde a temperatura máxima para ambos os casos foi de $1000^{\circ} \mathrm{C}$ e o padrão utilizado na DTA foi o óxido de alumínio $\left(\mathrm{Al}_{2} \mathrm{O}_{3}\right)$ calcinado, em atmosfera de ar [20,21].

A amostra AM1 teve suas propriedades reológicas estudadas no ano de 1995 [19] com base em norma da indústria do petróleo [22]. No ano de 2015, a amostra AM1 foi novamente estudada e comparada às propriedades reológicas no decorrer dos anos, visando à comparação com o estudo anterior [19]. As propriedades reológicas das amostras AM1 a $\mathrm{AM} 4$, industrialmente aditivadas com $\mathrm{Na}_{2} \mathrm{CO}_{3}$ no ano de 1995, foram comparadas com as propriedades reológicas das amostras AM5 a AM8, aditivadas em laboratório no ano de 2015. As amostras de argilas esmectíticas policatiônicas naturais AM5 a AM8 foram aditivadas com $\mathrm{Na}_{2} \mathrm{CO}_{3}$ nas mesmas proporções do ano de 1995, ou seja, 75, 100, 125 e $150 \mathrm{meq} / 100 \mathrm{~g}$, segundo a metodologia descrita em [20], onde foi escolhida a concentração de $125 \mathrm{meq} / 100 \mathrm{~g}$ de $\mathrm{Na}_{2} \mathrm{CO}_{3}$, correspondente aos melhores resultados entre as proporções citadas. Os valores de viscosidade aparente (VA), viscosidade plástica (VP) e volume do filtrado (VF) foram 
determinados conforme metodologias descritas em [23].

\section{RESULTADOS E DISCUSSÃO}

Na Fig. 1 estão apresentados os valores das distribuições granulométricas das amostras estudadas. Verificou-se que as amostras de argilas estudadas AM1 a AM8 apresentaram curvas de distribuição de partículas monomodais, observando-se uma maior concentração de partículas entre 0,5 e $20 \mu \mathrm{m}$ e ausência total ou parcial da fração com $\mathrm{x}>20$ $\mu \mathrm{m}$ (x - tamanho de partícula). A fração com $\mathrm{x}<2 \mu \mathrm{m}$ foi encontrada em quantidades maiores nas amostras de argilas esmectíticas sódicas quando comparadas com as amostras policatiônicas. As amostras apresentaram diferentes diâmetros médios, sendo maiores nas amostras de argilas esmectíticas policatiônicas, com valores entre 4,92 e 6,90 $\mu \mathrm{m}$

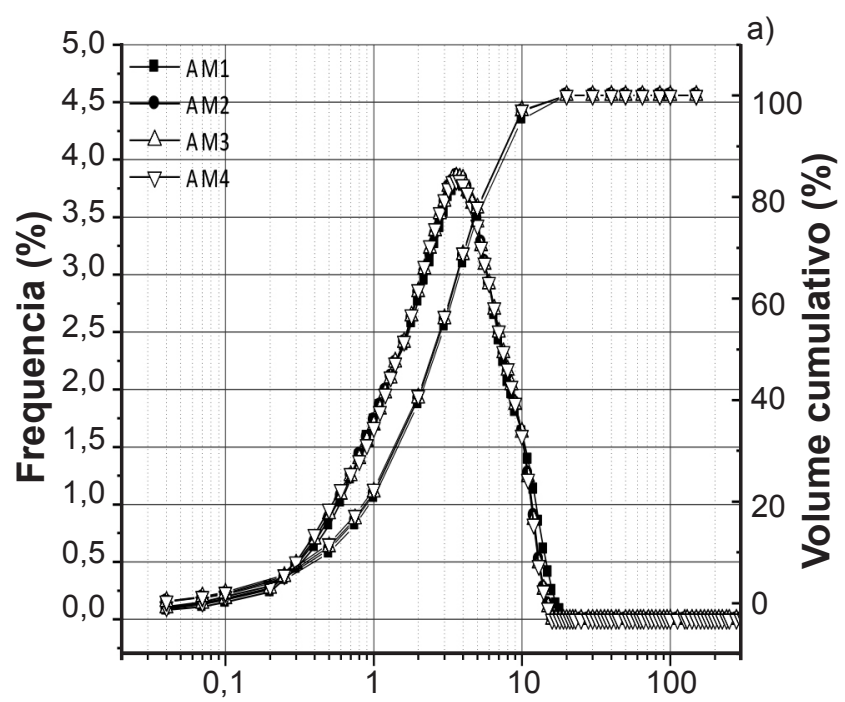

Tamanho de partícula $(\mu \mathrm{m})$

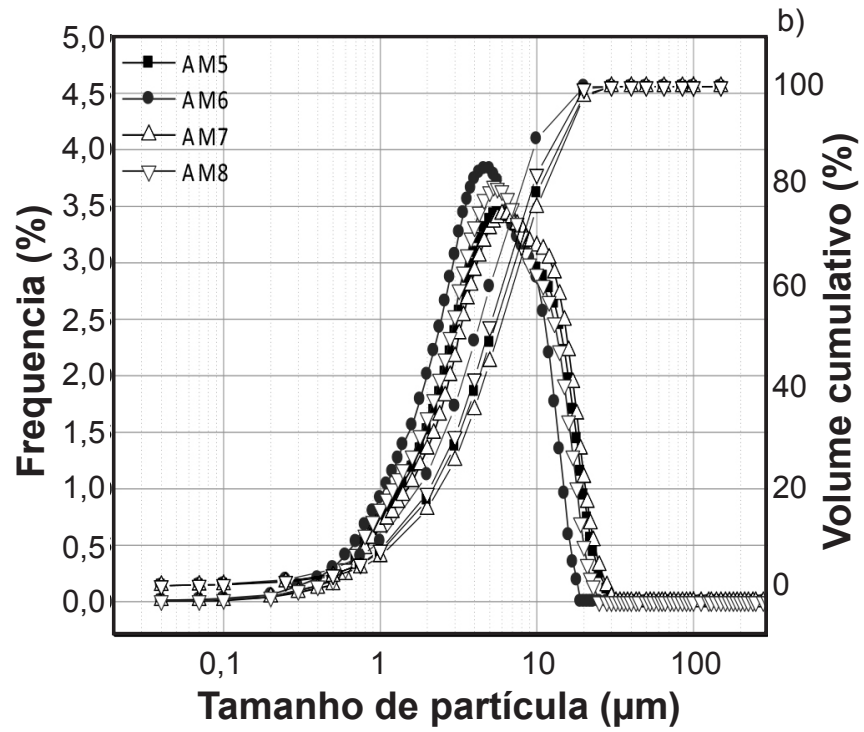

Figura 1: Distribuição granulométrica das amostras: (a) AM1 a AM4; (b) AM5 a AM8.

[Figure 1: Particle size distribution of samples: (a) AM1 to AM4; (b) AM5 to AM8.] e menores nas amostras de argilas esmectíticas sódicas, com valores entre 2,26 e 3,49 $\mu \mathrm{m}$; isso ressalta o fato das argilas sódicas apresentarem uma maior quantidade de fração com $\mathrm{x}<2 \mu \mathrm{m}$ e que conduz a melhores propriedades reológicas (conforme [14, 15], melhores propriedades reológicas são encontradas em amostras com menores diâmetros médios e com maiores teores de partículas com $\mathrm{x}<2 \mu \mathrm{m}$ ).

A Tabela I apresenta os teores de umidade (\%) das amostras com base na norma da ABNT [24] e de uma indústria petrolífera [25]. Os resultados dos teores de umidade que se baseiam na ABNT foram mais elevados por levar em consideração a massa seca da argila. Pode-se observar também que a umidade da AM1 foi de 17,23\% e $14,70 \%$ segundo a ABNT e a indústria petrolífera, respectivamente, mostrando assim um aumento de umidade quando comparada ao ano de 1995, onde não ultrapassava $14 \%$. A umidade especificada pela Petrobras não pode ultrapassar 14\% [25]. Umidades muito reduzidas podem ser responsáveis por propriedades reológicas inadequadas para fluidos de perfuração, principalmente quando os teores de $\mathrm{K}_{2} \mathrm{O}$ são mais elevados [26]. Segundo [27], quando o teor de umidade é inferior a $10 \%$, pode haver um aumento do volume de filtrado e redução nas viscosidades VA e VP.

A Tabela II apresenta a composição química das amostras em estudo. Verificou-se que as amostras apresentaram elevados teores de $\mathrm{SiO}_{2}$ e $\mathrm{Al}_{2} \mathrm{O}_{3}$ provenientes das camadas tetraédricas, sílica livre e outros minerais. Segundo [14, 15], baixos teores de sílica $\left(\mathrm{SiO}_{2}\right)$ e altos teores de alumina $\left(\mathrm{Al}_{2} \mathrm{O}_{3}\right)$ são indicativos do alto teor de argilominerais presentes; o oposto disso é indicativo da baixa proporção de minerais de argila e grandes quantidades de quartzo livre na amostra, como pode ser observado nos difratogramas de raios X (Fig. 2). A presença do $\mathrm{Fe}^{3+}, \mathrm{Mg}^{2+}$ e outros íons são decorrentes de substituições isomórficas que ocorrem na camada octaédrica da argila [28]. Para o teor de $\mathrm{Fe}_{2} \mathrm{O}_{3}$ observou-se que as amostras apresentaram teores entre 8 e $9 \%$, típicos das argilas esmectíticas [4, 28, 29]. Os óxidos alcalino-terrosos $\mathrm{CaO}$ e $\mathrm{MgO}$ apresentaram teores consideráveis, podendo ser uma indicação da presença de carbonatos $[14,15]$. Os valores de $\mathrm{K}_{2} \mathrm{O}$ e $\mathrm{TiO}_{2}$ apresentaramse relativamente baixos, inferiores a $1 \%$. Comparando os valores determinados, verificou-se que as amostras de

Tabela I - Teor de umidade (\% em massa) das amostras. [Table I - Moisture content (wt\%) of samples.]

\begin{tabular}{ccc}
\hline Amostra & ABNT [23] & Petrobras [24] \\
\hline AM1 & 17,23 & 14,70 \\
AM2 & 18,06 & 15,30 \\
AM3 & 17,37 & 14,80 \\
AM4 & 20,33 & 16,00 \\
AM5 & 21,20 & 17,90 \\
AM6 & 21,06 & 17,40 \\
AM7 & 19,31 & 16,20 \\
AM8 & 20,48 & 17,00 \\
\hline
\end{tabular}


Tabela II - Composição química (\% em massa) das amostras em estudo.

[Table II - Chemical composition (wt\%) of the studied samples.]

\begin{tabular}{ccccccccccc}
\hline Amostra & $\mathrm{SiO}_{2}$ & $\mathrm{Al}_{2} \mathrm{O}_{3}$ & $\mathrm{Fe}_{2} \mathrm{O}_{3}$ & $\mathrm{MgO}$ & $\mathrm{CaO}$ & $\mathrm{Na}_{2} \mathrm{O}$ & $\mathrm{K}_{2} \mathrm{O}$ & $\mathrm{TiO}_{2}$ & Outros & $\mathrm{PF}$ \\
\hline AM1 & 56,4 & 17,1 & 8,8 & 2,4 & 0,8 & 1,3 & 0,3 & 0,8 & 1,3 & 10,8 \\
AM2 & 57,6 & 17,3 & 8,7 & 2,5 & 0,8 & 1,2 & 0,3 & 0,9 & 0,7 & 9,9 \\
AM3 & 57,1 & 17,0 & 8,9 & 2,4 & 0,8 & 1,2 & 0,4 & 0,8 & 1,2 & 10,2 \\
AM4 & 58,7 & 17,5 & 9,3 & 2,4 & 0,8 & 1,3 & 0,3 & 0,8 & 1,3 & 8,7 \\
AM5 & 56,6 & 16,5 & 8,3 & 2,6 & 2,0 & - & 0,4 & 1,1 & 0,7 & 11,8 \\
AM6 & 55,5 & 16,0 & 8,6 & 2,5 & 1,9 & - & 0,4 & 0,9 & 1,3 & 12,9 \\
AM7 & 56,5 & 16,3 & 8,6 & 2,7 & 2,0 & - & 0,4 & 1,0 & 1,2 & 11,3 \\
AM8 & 56,2 & 16,2 & 8,7 & 2,6 & 2,0 & - & 0,4 & 1,0 & 1,2 & 11,7 \\
\hline
\end{tabular}

Notas: - ausente; PF: perda ao fogo.

argilas estudadas possuíram composições químicas típicas das argilas esmectíticas [4, 21].

A Fig. 2 apresenta os resultados de difração de raios $\mathrm{X}$ das amostras estudadas. Pôde-se observar as seguintes fases mineralógicas: esmectita, caracterizada por valores de espaçamento interplanar, d, de 15,20, 4,48 e 3,05 Å; quartzo, caracterizado por valores de d de 4,26, 3,34, 2,16, 1,81 e 1,67 $\AA$, compatível com os elevados teores de $\mathrm{SiO}_{2}$ das composições químicas das amostras (Tabela II). Na amostra AM2 pôde-se observar a fase mineralógica da caulinita, caracterizada por $\mathrm{d}=7,15 \AA$ [26]. Pôde-se observar também que a distância interplanar do pico característico da esmectita $\mathrm{d}_{(001)}$ foi semelhante para todas as amostras (entre 14,79 e $15,79 \AA$ ). As argilas esmectíticas estudadas anteriormente [2, 4, 20, 21] apresentaram as mesmas fases mineralógicas, sendo similares às argilas em estudo. $\mathrm{O}$ tratamento por

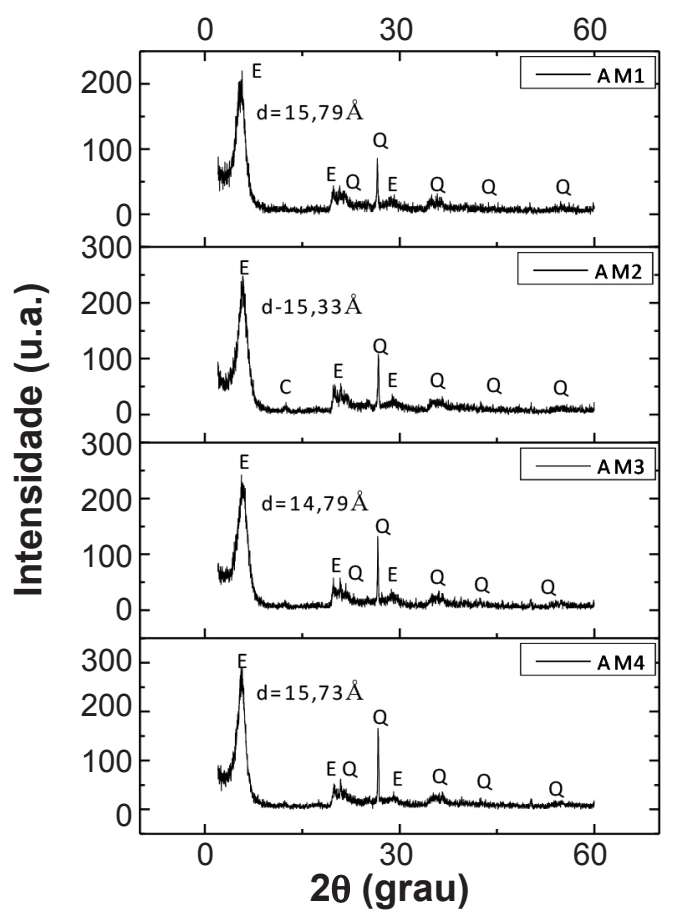

etileno glicol confirmou que todas as argilas estudadas foram esmectíticas, contudo, os difratogramas não foram incluídos neste trabalho.

A Fig. 3 apresenta as curvas termogravimétricas e termodiferenciais das argilas em estudo. Analisando as curvas de DTA das amostras (Figs. 3a e 3c) foram observadas as seguintes transformações térmicas: grande pico endotérmico em torno de $100{ }^{\circ} \mathrm{C}$, caracterizando a presença de água livre e adsorvida; banda exotérmica em torno de 180 a $450{ }^{\circ} \mathrm{C}$, correspondente à combustão de matéria orgânica; pico endotérmico em torno de $510{ }^{\circ} \mathrm{C}$ caracterizando a presença de hidroxilas da folha octaédrica; e pico endotérmico com máximo em $900{ }^{\circ} \mathrm{C}$ característico do carbonato de cálcio. Analisando as curvas de TG das amostras (Figs. 3b e 3d) observou-se que as perdas totais de massas das argilas esmectíticas sódicas foram de 22,32\%,

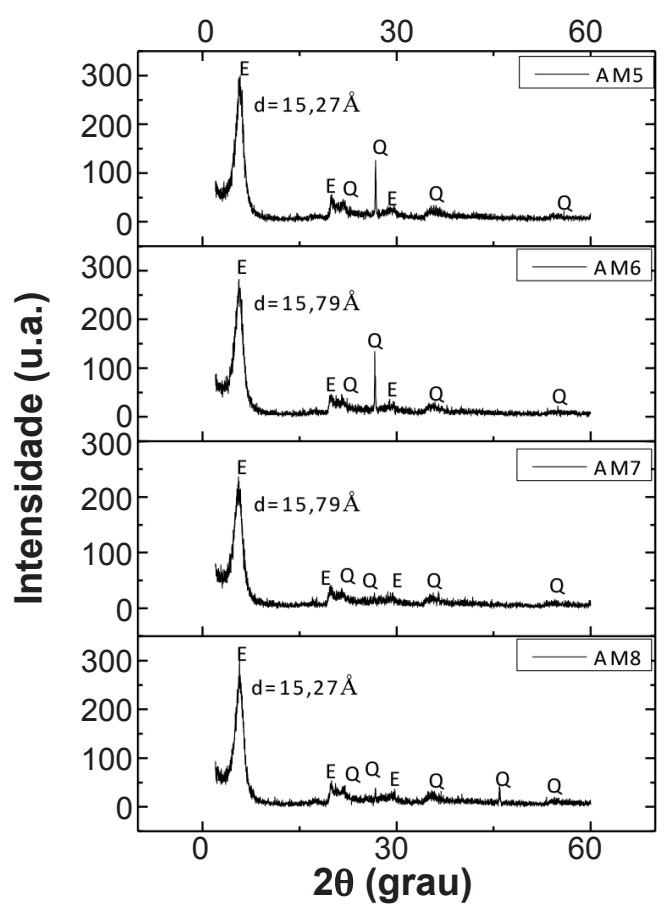

Figura 2: Difratogramas de raios X das amostras estudadas: (a) AM1 a AM4; (b) AM5 a AM8. E - esmectita; Q - quartzo. [Figure 2: X-ray diffraction patterns of samples: (a) AM1 to AM4; (b) AM5 to AM8. E - smectite; $Q$ - quartz.] 
$21,84 \%, 22,21 \%$ e $22,52 \%$ para as amostras AM1, AM2, AM3 e AM4, respectivamente, e as perdas totais de massas das argilas esmectíticas naturais foram de 26,95\%, 25,80\%, $24,22 \%$ e $25,46 \%$ para as amostras AM5, AM6, AM7e AM8, respectivamente, correspondente às perdas de água, de matéria orgânica, hidroxilas e carbonatos. Comparando os resultados dos termogramas em estudo com os encontrados em estudos anteriores [2, 4, 20, 21], observou-se um comportamento típico de argilas esmectíticas.

A Fig. 4 apresenta a comparação do comportamento reológico da amostra AM1 no ano de 1995 [19] com a mesma amostra AM1 no ano de 2015 utilizando parâmetros da norma de uma indústria petrolífera, vigente na época da pesquisa [19]. Analisando a Fig. 4, pode-se observar que a comparação dos resultados reológicos da amostra AM1/1995 e AM1/2015 evidencia uma melhora significativa nas propriedades reológicas ao longo dos anos. Em 1995 a dispersão não atingia as especificações estabelecidas pela indústria de petróleo, porém, no presente ano (2015), os valores de viscosidade aparente (VA) e viscosidade plástica (VP) aumentaram e o volume de filtrado (VF) diminuiu, características que comprovam a melhora das propriedades reológicas ao longo dos anos se enquadrando nas especificações da indústria petrolífera; já as propriedades reológicas da amostra em 1995 foram inferiores devido à reversibilidade das reações de dupla troca ao longo dos anos. No caso específico das amostras em estudo, os teores de umidade situaram-se na faixa de $14,80 \%$ a $17,90 \%$ (Tabela I), sendo mais elevados após o envelhecimento do que os especificados pela indústria do petróleo, cujo valor máximo é de $14 \%$, fazendo o aumento da umidade outro fator a favor para essa melhora. Outro fator determinante para isto foi o armazenamento/acondicionamento adequado para as argilas ao longo dos anos (sacos fechados), contribuindo assim para que, possivelmente, reações de troca se completassem com o decorrer do tempo. Segundo [30], as argilas sódicas são mais fortemente hidratadas, tendo um inchamento mais significativo das partículas causando assim um grande aumento em suas propriedades reológicas, o que é reforçado pelo envelhecimento, pelo aumento da umidade. $\mathrm{O}$ aumento do teor de umidade durante o envelhecimento melhora a cinética da reação entre o $\mathrm{Na}_{2} \mathrm{CO}_{3}$ e as argilas, resultando
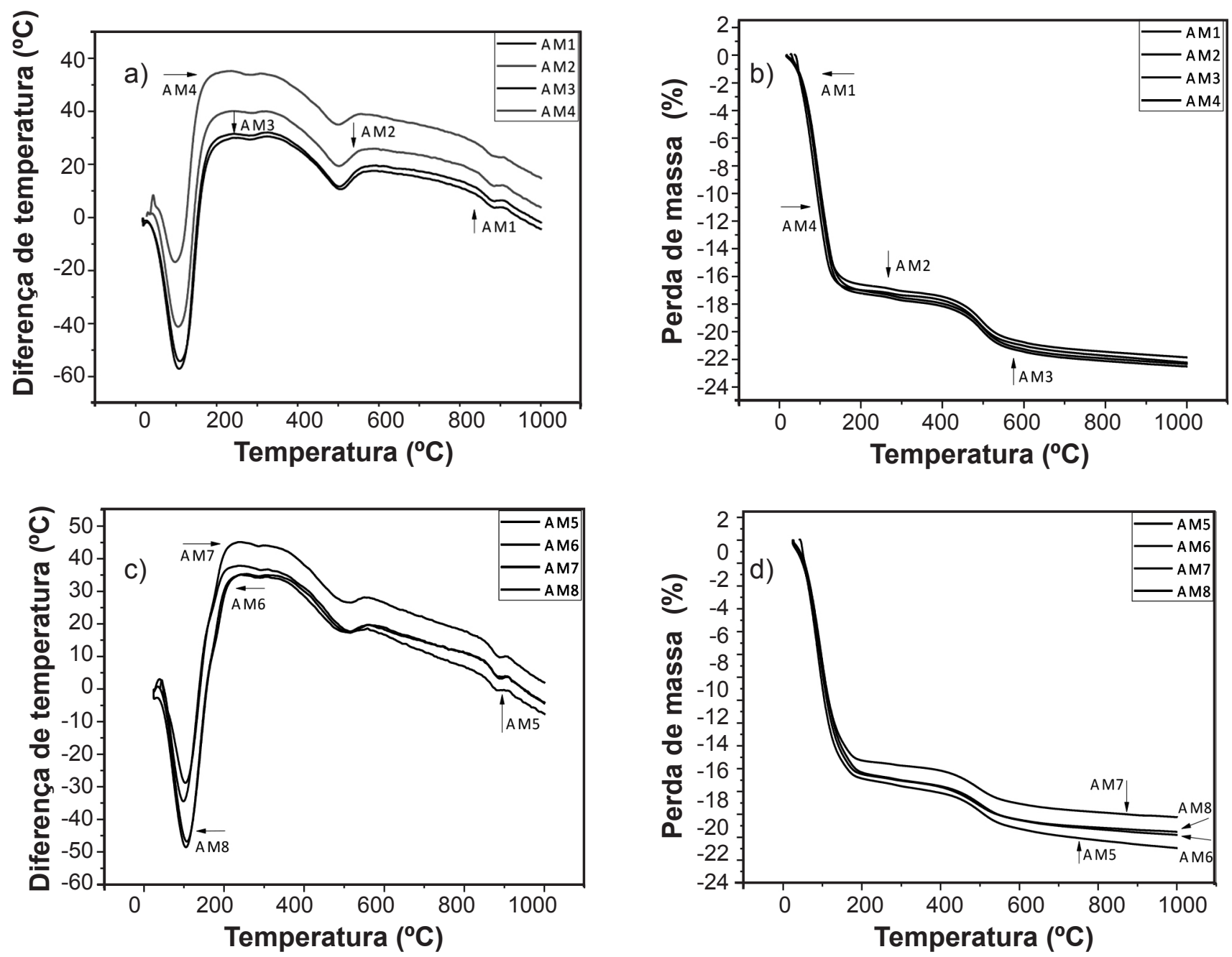

Figura 3: Curvas termodiferenciais (a, c) e termogravimétricas (b, d) das amostras estudadas.

[Figure 3: Thermodifferential $(a, c)$ and thermogravimetric $(b, d)$ curves of the samples.] 


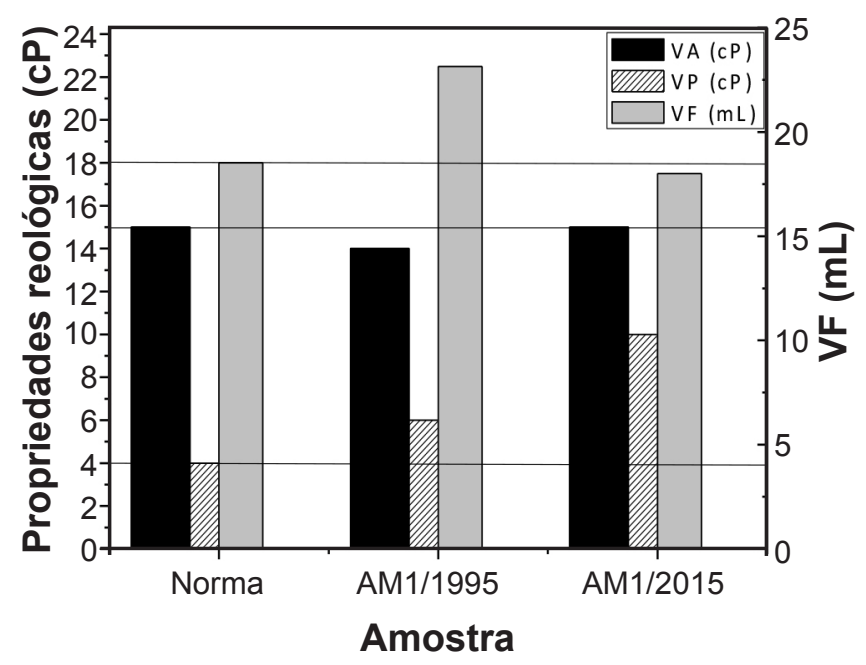

Figura 4: Propriedades reológicas da AM1/1995 e AM1/2015. [Figure 4: Rheological properties of AM1/1995 and AM1/2015.]

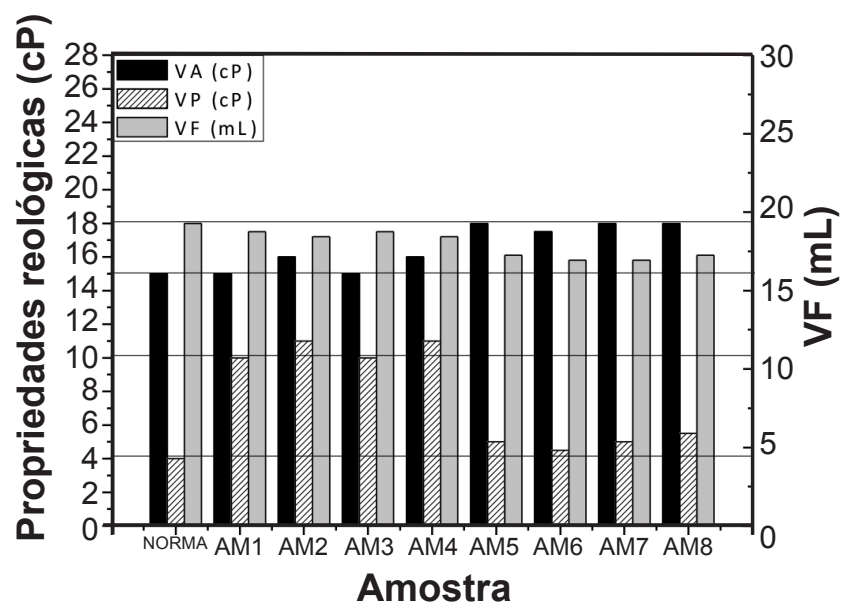

Figura 5: Propriedades reológicas das amostras AM1 a AM4 aditivadas industrialmente em 1995 e AM5 a AM8 aditivadas em laboratório no ano de 2015.

[Figure 5: Rheological properties of the samples AM1 to AM4 doped industrially in 1995, and AM5 to AM8 additivated in the laboratory in 2015.]

no aumento da VA e VP e diminuição do VF $[16,18,31]$. Estudos de $[8,31]$ também confirmam o efeito benéfico dos elevados teores de umidade na cinética da reação de troca de cátions que ocorre entre $\mathrm{o} \mathrm{Na}_{2} \mathrm{CO}_{3}$ e os policátions existentes nas esmectitas naturais.

A Fig. 5 apresenta os resultados reológicos das amostras de argilas esmectíticas AM1 a AM4 aditivadas industrialmente no ano de 1995 e as amostras AM5 a AM8 aditivadas em laboratório com $125 \mathrm{meq} / 100 \mathrm{~g}$ de $\mathrm{Na}_{2} \mathrm{CO}_{3}$ no ano de 2015. Analisando a Fig. 5, pode-se observar que as amostras aditivadas com $125 \mathrm{meq} / 100 \mathrm{~g}$ de $\mathrm{Na}_{2} \mathrm{CO}_{3}$ no ano de 2015 (AM5 a AM8) apresentaram valores de VA maiores do que as amostras aditivadas industrialmente no ano 1995 (AM1 a AM4), devido à reversibilidade das reações de troca de cátions no decorrer dos anos. As amostras AM1 a AM4 apresentaram valores de VP maiores que as aditivadas em
2015 (AM5 a AM8); isto se deveu provavelmente, ao fato de que o processo de aditivação de argilas industrialmente é mais eficaz do que a aditivação efetuada em laboratório, uma vez que, em laboratório, esse processo não leva em consideração a adição do $\mathrm{Na}_{2} \mathrm{CO}_{3} \mathrm{em}$ pó, perdendo com isso a temperatura da reação exotérmica com a adição da água, e não tem a etapa da laminação e extrusão com adição de vapor, além da secagem, que nas indústrias de maior porte é feita com secadores a vácuo, fazendo com que o $\mathrm{Na}_{2} \mathrm{CO}_{3}$ tenha um menor contato com a esmectita. Além disso, a secagem em laboratório é feita em estufa com temperaturas superiores a $60{ }^{\circ} \mathrm{C}$, influenciando diretamente nas propriedades reológicas da argila $[6,26,27]$. Outro fator que contribui para esses resultados é que as amostras aditivadas industrialmente apresentaram maiores valores de fração $\mathrm{x}<2 \mu \mathrm{m}$ e menores diâmetros médios (Fig. 1) que as amostras policatiônicas, comprovando que melhores propriedades reológicas são encontradas em amostras com menores diâmetros médios e com maiores teores de partículas com $\mathrm{x}<2 \mu \mathrm{m}[14,15]$.

\section{CONCLUSÕES}

Com base nesse estudo, pôde-se concluir que houve uma melhora significativa nas propriedades reológicas das dispersões das amostras de argilas esmectíticas após os anos de envelhecimento. Isso se deveu, sobretudo, a não reversibilidade das reações de dupla troca ao longo dos anos, armazenamento/acondicionamento adequado e aumento da umidade; pôde-se concluir também que houve influência da quantidade de fração argila e de diferentes processos de ativação ao comparar amostras aditivadas industrialmente com as amostras policatiônicas aditivadas em laboratório ao longo dos anos. Esses estudos também comprovaram que validades inferiores a 1 ano são excessivamente reduzidas, pois amostras devidamente acondicionadas e armazenadas não apresentaram perda de propriedades indicando a não reversibilidade das reações de dupla troca quando da transformação das argilas policatiônicas em sódicas.

\section{REFERÊNCIAS}

[1] K. Choo, K. Bai, "Effects of bentonite concentration and solution $\mathrm{pH}$ on the rheological properties and long-term stabilities of bentonite suspensions", Appl. Clay Sci. 108 (2015) 182.

[2] R.R. Menezes, P.M. Souto, L.N.L. Santana, G.A. Neves, R.H.G.A. Kiminami, H.C. Ferreira, "Argilas bentoníticas de Cubati, Paraíba, Brasil: caracterização físicamineralógica", Cerâmica 55, 334 (2009) 163.

[3] R.R. Menezes, L.N. Marques, L.A. Campos, H.C. Ferreira, L.N.L. Santana, G.A. Neves, "Use of statistical design to study the influence of CMC on the rheological properties of bentonite dispersions for water-based drilling fluids", Appl. Clay Sci. 49 (2010) 13.

[4] H.S. Ferreira, L.F.A. Campos, R.R. Menezes, J.M. Cartaxo, L.N.L. Santana, G.A. Neves, H.C. Ferreira, "Influência das variáveis de processo na obtenção de argilas 
organofílicas", Cerâmica 59, 350 (2013) 277.

[5] P. Skalle, Drilling fluid engineering, Pal Skalle - Ventus Publishing ApS, Dept. Petrol. Eng. Appl. Geophys., NTNU, Trondheim (2011) 23.

[6] M.S. El-Mahllawy, A.M. Sharara, M.M. Hassaan, A.M.A. Haleem, "The composition and activation aspects of El-Fayoum clays for using as a drilling fluid", Egyptian J. Petroleum 22 (2013) 395.

[7] A. Nosrati, J. Addai-Mensah, W. Skinner, "Rheology of aging aqueous muscovite clay dispersions", Chem. Eng. Sci. 66 (2011) 119.

[8] C. Karagüzel, T. Çetinel, F. Boylu, K. Çinku, M.S. Çelik, "Activation of ( $\mathrm{Na}, \mathrm{Ca}$ )-bentonites with soda and $\mathrm{MgO}$ and their utilization as drilling mud", Appl. Clay Sci. 48 (2010) 398.

[9] F. Shayeganfar, M. SadeghMovahed, G.R. Jafari, "Discrimination of sol and gel states in an aging clay suspension", Chem. Phys. 423 (2013) 167.

[10] C. Liang, W. Sun, T. Wang, X. Liu, Z. Tong, "Rheological inversion of the universal aging dynamics of hectorite clay suspensions", Colloids Surf., A: Physicochem. Eng. Aspects 490 (2016) 300.

[11] F. Boylu, "Modelling and optimization of ageing characteristics of soda activated $\mathrm{Na}^{+}$-bentonites", Appl. Clay Sci. 83 (2013) 300.

[12] K. Sehly, H.L. Chiew, H. Li, A. Song, Y.K. Leong, W. Huang, "Stability and ageing behaviour and the formulation of potassium-based drilling muds", Appl. Clay Sci. 104 (2015) 309.

[13] R.H.G.A. Kiminami, E.C.C. Machado, H.C. Ferreira, "Propriedades reológicas das argilas montmoriloníticas do distrito de Boa Vista - Campina Grande, PB, após tratamento com carbonato de sódio e cura em câmara climatizada visando sua aplicação em fluidos de perfuração de poços de petróleo", Cerâmica 26 (1984) 307.

[14] R. Gaidzinski, P. Osterreicher-Cunha, J. Duailibi Fh., L.M. Tavares, "Modification of clay properties by aging: role of indigenous microbiota and implications for ceramic processing", Appl. Clay Sci. 43 (2009) 98.

[15] R. Gaidzinski, J. Duailibi Fh., L.M. Tavares, "Influence of aging on the technological properties of clays in the ceramic industry", Appl. Clay Sci. 54 (2011) 47.

[16] R. Shu, W. Sun, X. Liu, Z. Tong, "Temperature dependence of aging kinetics of hectorite clay suspensions", J. Colloid Interface Sci. 444 (2015) 132.

[17] S.J. He, Y.Q. Wang, M.M. Xi, J. Lin, Y. Xue, L.Q. Zhang, "Prevention of oxide aging acceleration by nanodispersed clay in styrene-butadiene rubber matrix", Polym. Degrad. Stab. 98 (2013) 1773.
[18] C.O. Modesto, A.M. Bernardin, "Determination of clay plasticity: indentation method versus Pfefferkorn method", Appl. Clay Sci. 40 (2008) 15.

[19] H.S.Ferreira, G.A.Neves, H.C.Ferreira, "Sensibilização de dispersões de esmectíticas pelo uso do CMC", Anais do $40^{\circ}$ Congresso Brasileiro de Cerâmica (1995).

[20] I.A. Silva, J.M.R. Costa, R.R. Menezes, H.S. Ferreira, G.A. Neves, H.C. Ferreira, "Studies of new occurrences of bentonite clays in the State of Paraíba for use in water based drilling fluids", Rev. Escola Minas 66 (2013) 485.

[21] I.A. Silva, F.K.A. Sousa, R.R. Menezes, G.A. Neves, L.N.L. Santana, H.C. Ferreira, "Modification of bentonites with nonionic surfactants for use in organic-based drilling fluids", Appl. Clay Sci. 95 (2014) 371.

[22] Petrobras, "Argila ativada para água doce", EE-78 (1968).

[23] B.M.A. Brito, J.M. Cartaxo, N.F.C. Nascimento, H.C. Ferreira, G.A. Neves, R.R. Menezes, "Avaliação de argilas bentoníticas policatiônicas do estado da Paraíba com aditivos para aplicação em perfuração de poços de petróleo e tintas à base de água", Cerâmica 62, 361 (2016) 45-54.

[24] ABNT - Associação Brasileira de Normas Técnicas, NBR 6457, "Amostras de solo - Preparação para ensaios de compactação e ensaios de caracterização" (1986).

[25] Petrobras, "Ensaio de viscosificante para fluidos base água na exploração e produção de petróleo", Código: EP1EP-00011-A (2011).

[26] P. Souza Santos, Tecnologia de argilas, Edgard Blücher, S. Paulo (1992).

[27] E. Teixeira-Neto, A.A. Teixeira-Neto, "Modificação química de argilas: desafios científicos e tecnológicos para obtenção de novos produtos com maior valor agregado", Quim. Nova 32 (2009) 809.

[28] C. Bertagnolli, M.G.C. Silva, "Characterization of Brazilian bentonite organoclays as sorbents of petroleumderived fuels", Mater. Res. 15 (2012) 253.

[29] I.A. Silva, J.M.R. Costa, H.S. Ferreira, R.R. Menezes, G.A. Neves, H.C. Ferreira, "Organofilização de argilas bentoníticas com tensoativos não-iônicos visando seu uso em fluidos de perfuração base óleo", Cerâmica 58, 347 (2012) 317.

[30] W.Z. Chang, Y.K. Leong, "Ageing and collapse of bentonite gels - effects of $\mathrm{Li}, \mathrm{Na}, \mathrm{K}$ and Cs ions", Rheologic Acta 53 (2014) 109.

[31] A.R. Zandonadi, "Estudo tecnológico de argilas montimoriloníticas brasileiras", Tese Dr., Instituto de Química da USP, S. Paulo, SP (1972).

(Rec. 11/03/2016, Rev. 25/05/2016, 20/07/2016, Ac. 20/07/2016) 